\title{
KEYNOTE-042 rolls back programmed cell death ligand 1 threshold for non-small cell lung cancer pembrolizumab monotherapy without new insight into those deriving benefit
}

\author{
Amy Lauren Cummings, Edward B. Garon \\ Department of Medicine, Division of Hematology/Oncology, David Geffen School of Medicine at the University of California, Los Angeles, \\ CA, USA \\ Correspondence to: Edward B. Garon, MD, MS. Associate Professor, Department of Medicine, Division of Hematology/Oncology, David \\ Geffen School of Medicine at the University of California, Los Angeles, 2825 Santa Monica Blvd, Suite 220, Santa Monica, CA 90404, USA. \\ Email: egaron@mednet.ucla.edu. \\ Provenance: This is an invited article commissioned by the Section Editor Jun Zhou, MD (Department of Nuclear Medicine, Zhongshan Hospital, \\ Fudan University, Shanghai, China). \\ Comment on: Mok TSK, Wu YL, Kudaba I, et al. Pembrolizumab versus chemotherapy for previously untreated, PD-L1-expressing, locally advanced \\ or metastatic non-small-cell lung cancer (KEYNOTE-042): a randomised, open-label, controlled, phase 3 trial. Lancet 2019;393:1819-30.
}

Submitted Jul 11, 2019. Accepted for publication Jul 18, 2019.

doi: 10.21037/tlcr.2019.07.06

View this article at: http://dx.doi.org/10.21037/tlcr.2019.07.06

The past two years have been exceptional for pembrolizumab in advanced non-small cell lung cancer (NSCLC). The publication of KEYNOTE-189 redefined first-line treatment with combination chemotherapy and pembrolizumab as a new standard-of-care in an unselected population, KEYNOTE-042 expanded the United States Food \& Drug Administration (FDA)'s approval for first-line pembrolizumab monotherapy, and KEYNOTE-001 showed the most robust five-year overall survival (OS) results ever seen in advanced NSCLC, specifically among patients with high programmed cell death ligand 1 (PD-L1) expression (Table 1) (1-4). As we have suggested previously, KEYNOTE trials have benefited from being carefully tailored with preplanned analyses with
PD-L1 immunohistochemistry (IHC) tumor proportion score (TPS) cutoffs using the $22 \mathrm{C} 3$ pharmDx assay (Agilent Technologies, Carpinteria, CA, USA) $(5,6)$. Yet while these trials suggest that PD-L1 TPS $\geq 50 \%$ remains the most reliable biomarker for durable response with pembrolizumab, what to do with PD-L1 TPS scores less than 50\% continues to be challenging. Does a $1 \%$ difference really change clinical outcome?

KEYNOTE-042 attempted to answer this question in the case of pembrolizumab monotherapy compared to standard chemotherapy (2). Randomizing over 1,200 patients with PD-L1 TPS $\geq 1 \%$ in 213 medical centers in 32 countries around the world, it is one of the largest studies

Table 1 Overall survival median by PD-L1 22C3 assay cutoffs

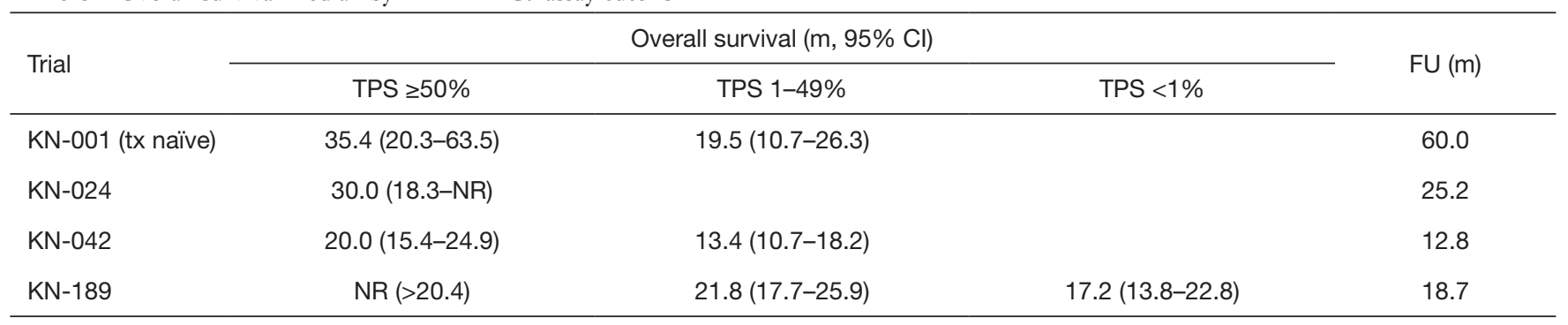

$\mathrm{Cl}$, confidence interval; FU, median follow-up; KN, KEYNOTE; m, month(s); NR, not reached; PD-L1, programmed cell death ligand 1; TPS, tumor proportion score; tx, treatment. 
of pembrolizumab to date. Its primary endpoint was OS with planned analyses of three groups based on PD-L1 TPS, including $\geq 50 \%, \geq 20 \%$, and $\geq 1 \%$ cutoffs, requiring a split alpha and $\mathrm{P}$ value of $0.0122,0.0120$, and 0.0124 to meet statistical one-sided significance. Remarkably, the study met all of these endpoints-for the $\geq 50 \%$ group, OS median was 20.0 months [ $95 \%$ confidence interval (CI), 15.4-24.9 months] with a hazard ratio for death (HR) of 0.69 (95\% CI, $0.56-0.85$ ); for $\geq 20 \%$, OS was 17.7 months (95\% CI, 15.3-24.9 months) with a HR of 0.77 (95\% CI, $0.64-0.92$ ); and for $\geq 1 \%$, OS was 16.7 months (95\% CI, 13.9-19.7 months) with a HR of 0.81 (95\% CI, 0.71-0.93). This is impressive, but it should be noted that patients with a PD-L1 TPS $\geq 50 \%$ contributed to the favorable profile of each evaluated group. The 299 participants treated with single-agent pembrolizumab with a PD-L1 TPS $\geq 50 \%$ comprised $72.4 \%$ of the 413 patients in the $\geq 20 \%$ group and $46.9 \%$ of the 637 patients in the $\geq 1 \%$ group.

As an exploratory endpoint, only those with a PD-L1 TPS 1-49\% were evaluated for OS, including 338 treated with pembrolizumab and 337 treated with chemotherapy. OS was 13.4 months (95\% CI, 10.7-18.2 months) with pembrolizumab versus 12.1 months with chemotherapy (95\% CI, 11.0-14.0), which did not meet statistical significance with a HR of 0.92 (95\% CI, 0.77-1.11). Further, progression-free survival (PFS) was not significantly different at any TPS cutoff. While PD-L1 TPS $\geq 50 \%$ did perform better than $\geq 20 \%$ and $\geq 1 \%$, median PFS was 7.1 months (95\% CI, 5.9-9.0 months) versus 6.4 months (95\% CI, 6.1-6.9 months) with a HR of 0.81 (95\% CI, 0.67-0.99; $\mathrm{P}=0.0170$ ), which did not meet its prespecified $P$ value. PFS was not evaluated in the $1-49 \%, \geq 20 \%$, or $\geq 1 \%$ groups as a result. Overall response rate was similarly not statistically significant between any arms in any population.

The discordance of these secondary and exploratory endpoints is problematic to KEYNOTE-042's narrative, particularly with respect to the PD-L1 TPS 1-49\% group, which received the FDA's PD-L1 expansion for pembrolizumab monotherapy. It is unlikely that the chemotherapy arm overperformed with an OS of 12.1 months, which tracks with what we would expect for the comparator regimens $(7,8)$. So did pembrolizumab underperform? Based on KEYNOTE-001, we would expect an OS median of around 18 months in treatment naïve patients with a PD-L1 TPS 1-49\%, and KEYNOTE-042 fell short of this mark by over 6 months. For PD-L1 TPS $\geq 50 \%$, KEYNOTE-024 had an OS of 30.0 months $(95 \%$ CI, 18.3-not reached), similar to the KEYNOTE-001's
OS in treatment-naïve patients of 35.4 months (95\% CI, 20.3-63.5 months), both of which are close to a year longer than KEYNOTE-042's OS median of 20.0 months (95\% CI, 15.4-24.9 months) (4,9).

As a possible explanation for pembrolizumab's lackluster KEYNOTE-042 performance, the authors note that unlike KEYNOTE-001 and KEYNOTE-024, KEYNOTE-042 enrolled a higher proportion of patients in east Asia (29\% vs. $0 / 13 \%)(2,4,9)$. Regional characteristics of KEYNOTE-042 support that for both the pembrolizumab and chemotherapy groups, east Asian patients comprised the largest number of participants in each of the TPS categories $(29-31 \%)$. While this population is known for a greater prevalence of epidermal growth factor receptor (EGFR) mutations, EGFR and $A L K$ testing were required, so it cannot be argued that there was an enrichment for patients with poor predictive markers (10-12). Further, while some small, retrospective analyses have suggested increased toxicity with checkpoint inhibition in east Asian populations, there were few dose-limiting toxicities in KEYNOTE-042, and toxicity has not been associated worse OS $(13,14)$. Yet one other consideration for KEYNOTE042's results as they relate to an east Asian population is PD-L1 expression. PD-L1 was assessed at two central laboratories, one for China and one for the rest of the world. While it is unlikely there were significant differences in how the PD-L1 22C3 assay was interpreted, there has been one study that has suggested that PD-L1 performs differently in east Asian NSCLC patients, noting that a TPS cutoff of $50 \%$ does not associate with significant differences in OS and that a low proportion of east Asian smokers may explain the discrepancy (15). KEYNOTE-042 has more than double the proportion of never-smokers at $22 \%$ compared to $11 \%$ in KEYNOTE- 001 and $3.2 \%$ in the pembrolizumab arm of KEYNOTE-024. As smoking has been associated with durable benefit in both nivolumab and pembrolizumab studies $(4,16)$, this is the most likely explanation for KEYNOTE-042's underperformance.

The KEYNOTE-042 authors also suggest that differences in crossover could have resulted in OS differences. In KEYNOTE-024, 64\% of participants in the chemotherapy arm ultimately received pembrolizumab, while crossover was not permitted in KEYNOTE-042. The authors suggest that in KEYNOTE-042 regions, there is less availability of and access to therapy, leading only $20 \%$ of the chemotherapy group to receive subsequent immunotherapy. While this would likely favor the pembrolizumab group, suboptimal treatment may extend 
to other types of modulating therapies like palliative radiation, which has been shown to benefit survival with pembrolizumab (17).

So, does KEYNOTE-042 convince us that pembrolizumab monotherapy in patients with PD-L1 TPS $1-49 \%$ is a comparable alternative to KEYNOTE189 's combination? We'd say not. Although cross-trial comparisons are frowned upon, KEYNOTE-189's OS median of 21.8 months is comfortably beyond the upper confidence limit of KEYNOTE-042's OS. But what should not be missed in KEYNOTE-042's PD-L1 TPS 1-49\% group is the notable difference in confidence intervals between the chemotherapy and pembrolizumab arms. The chemotherapy group had an interval of 11.0-14.0 months while pembrolizumab's was 10.7-18.2 months. Undoubtedly, there are individuals benefiting from pembrolizumab in this group. And while we ultimately hope to develop biomarkers to help us find these individuals among the PD-L1 TPS $1-49 \%$ group, we simply aren't there yet.

In this sense, KEYNOTE-042 enabled FDA approval of pembrolizumab at any positive level of PD-L1 expression. Even though pembrolizumab alone might not be as good as pembrolizumab with chemotherapy, in light of KEYNOTE-042's robust safety data with only $9 \%$ of patients experiencing an event leading to discontinuation of treatment and very few treatment-related deaths, there is an acceptable risk for a potentially real benefit. While the trial did not include patients with borderline ECOGs or those who refuse chemotherapy, these patients exist, and they are the ones that often slip through the cracks without ever receiving treatment. So, while 13.4 months may not beat 21.8 months, it more than doubles the 6 months that you would expect for someone without any appropriate treatment. Thus, while KEYNOTE-042 has not convinced us that pembrolizumab monotherapy is more appropriate than a combination of chemotherapy with pembrolizumab in patients with a PD-L1 TPS $1-49 \%$, it has given us the ability to access this agent for those who may otherwise not get treatment.

\section{Acknowledgments}

None.

\section{Footnote}

Conflicts of Interest: The authors have no conflicts of interest to declare.
Ethical Statement: The authors are accountable for all aspects of the work in ensuring that questions related to the accuracy or integrity of any part of the work are appropriately investigated and resolved.

\section{References}

1. Gandhi L, Rodriguez-Abreu D, Gadgeel S, et al. Pembrolizumab plus Chemotherapy in Metastatic NonSmall-Cell Lung Cancer. N Engl J Med 2018;378:2078-92.

2. Mok TSK, Wu YL, Kudaba I, et al. Pembrolizumab versus chemotherapy for previously untreated, PD-L1expressing, locally advanced or metastatic non-small-cell lung cancer (KEYNOTE-042): a randomised, open-label, controlled, phase 3 trial. Lancet 2019;393:1819-30.

3. United States Food and Drug Administration. FDA expands pembrolizumab indication for first-line treatment of NSCLC (TPS $\geq 1 \%$ ). Available online: https://www.fda. gov/drugs/fda-expands-pembrolizumab-indication-firstline-treatment-nsclc-tps-1. Accessed 25 June 2019.

4. Garon EB, Hellmann MD, Rizvi NA, et al. Five-Year Overall Survival for Patients With Advanced NonSmallCell Lung Cancer Treated With Pembrolizumab: Results From the Phase I KEYNOTE-001 Study. J Clin Oncol 2019;37:2518-27.

5. Cummings AL, Garon EB. The ascent of immune checkpoint inhibitors: is the understudy ready for a leading role? Cancer Biol Med 2017;14:341-7.

6. Garon EB, Rizvi NA, Hui R, et al. Pembrolizumab for the treatment of non-small-cell lung cancer. N Engl J Med 2015;372:2018-28.

7. Paz-Ares LG, de Marinis F, Dediu M, et al. PARAMOUNT: Final overall survival results of the phase III study of maintenance pemetrexed versus placebo immediately after induction treatment with pemetrexed plus cisplatin for advanced nonsquamous non-small-cell lung cancer. J Clin Oncol 2013;31:2895-902.

8. Sandler A, Gray R, Perry MC, et al. Paclitaxel-carboplatin alone or with bevacizumab for non-small-cell lung cancer. N Engl J Med 2006;355:2542-50.

9. Reck M, Rodriguez-Abreu D, Robinson AG, et al. Updated Analysis of KEYNOTE-024: Pembrolizumab Versus Platinum-Based Chemotherapy for Advanced NonSmall-Cell Lung Cancer With PD-L1 Tumor Proportion Score of 50\% or Greater. J Clin Oncol 2019;37:537-46.

10. Gainor JF, Shaw AT, Sequist LV, et al. EGFR Mutations and ALK Rearrangements Are Associated with Low Response Rates to PD-1 Pathway Blockade in Non-Small 
Cell Lung Cancer: A Retrospective Analysis. Clin Cancer Res 2016;22:4585-93.

11. Lisberg A, Cummings A, Goldman JW, et al. A Phase II Study of Pembrolizumab in EGFR-Mutant, PD-L1+, Tyrosine Kinase Inhibitor Naive Patients With Advanced NSCLC. J Thorac Oncol 2018;13:1138-45.

12. Kohno T, Nakaoku T, Tsuta K, et al. Beyond ALK-RET, ROS1 and other oncogene fusions in lung cancer. Transl Lung Cancer Res 2015;4:156-64.

13. Peng $\mathrm{L}, \mathrm{Wu} \mathrm{YL}$. Immunotherapy in the Asiatic population: any differences from Caucasian population? J Thorac Dis 2018;10:S1482-93.

14. Lisberg A, Tucker DA, Goldman JW, et al. TreatmentRelated Adverse Events Predict Improved Clinical Outcome in NSCLC Patients on KEYNOTE-001 at a

Cite this article as: Cummings $\mathrm{AL}$, Garon EB. KEYNOTE-042 rolls back programmed cell death ligand 1 threshold for non-small cell lung cancer pembrolizumab monotherapy without new insight into those deriving benefit. Transl Lung Cancer Res 2019;8(Suppl 4):S403-S406. doi: 10.21037/tlcr.2019.07.06
Single Center. Cancer Immunol Res 2018;6:288-94.

15. Pan Y, Zheng D, Li Y, et al. Unique distribution of programmed death ligand 1 (PD-L1) expression in East Asian non-small cell lung cancer. J Thorac Dis 2017;9:2579-86.

16. Gettinger S, Horn L, Jackman D, et al. Five-Year FollowUp of Nivolumab in Previously Treated Advanced NonSmall-Cell Lung Cancer: Results From the CA209-003 Study. J Clin Oncol 2018;36:1675-84.

17. Shaverdian N, Lisberg AE, Bornazyan K, et al. Previous radiotherapy and the clinical activity and toxicity of pembrolizumab in the treatment of non-small-cell lung cancer: a secondary analysis of the KEYNOTE-001 phase 1 trial. Lancet Oncol 2017;18:895-903. 